\title{
NUMERICAL INVESTIGATION ON THE EFFECTS OF SINGLE-MODE MICROWAVE TREATMENT ON ROCK BREAKAGE SYSTEM
}

\author{
K. Teimoori ${ }^{1}$, F. Hassani ${ }^{1}$, A.P. Sasmito ${ }^{1}$, and S.A. Ghoreishi-Madiseh ${ }^{2}$ \\ ${ }^{1}$ Department of Mining and Materials Engineering, McGill University, 3450 University, \\ Frank Dawson Adams Bldg., Montreal, QC H3A OE8, Canada \\ ${ }^{2}$ Norman B Keevil Institute of Mining Engineering, University of British Colombia, \\ Vancouver, BC, V6T 1Z4, Canada \\ khashayar.teimoori@mail.mcgill.ca \\ ferri.hassani@mcgill.ca \\ agus.sasmito@mcgill.ca \\ ali.madiseh@ubc.ca
}

Keywords: microwave heating, rock preconditioning, single-mode microwave treatment, Maxwell's theory

\begin{abstract}
In this study, a rock model which consists of a conceptual block (host rock and ore sample) is numerically modeled by using the finite element method. The rock model is subjected to several single-mode microwave treatments with different power levels, distances from the antenna, and exposure times in order to calculate and compare the corresponding effects including temperature distribution and mechanical stress/damage profiles. The main objective of the present study is to analyze the distribution of temperature and mechanical stress at the boundary of two different attached rocks when exposed to microwaves. This enables comparing the intensity of the distribution with respect to the applied microwave input operating parameters and, consequently, understanding rock preconditioning. The results of the present study verify that an increase in temperature by microwave treatment facilitates the rock weakening process. Also, a more efficient selection of the distance from the antenna and the power level can maximize the overall impact of the microwave treatment on rock preconditioning which ultimately helps with the rock breakage mechanism.
\end{abstract}

\section{Introduction}

The application of microwave heating has been investigated to precondition rocks prior to breakage by mechanical means such as a tunneling machine. The concept is that the microwave irradiation will heat the rock. The rock consists of different minerals with different thermal expansions at different rates, causing microcracks in the boundary of the minerals upon heating. Such microcracks consequently reduce the rock strength. This reduction will contribute to a higher penetration rate of the cutting machine and a reduction of wear on the cutting discs.

The technology of microwave-induced rock breakage has been considered a potential method for rock fracturing. The idea of applying microwaves to rocks began with a patented study conducted by Lindroth et al. in 1991. This process is referred to as "rock preconditioning" [1-3]. Fig. 1 illustrates the idea of microwave-assisted rock breakage as a potential alternative to conventional methods.

Understanding the complex process of rock breakage under microwave treatment requires a knowledge of the fundamental principles of electromagnetic waves, heat transfer theory, thermo-mechanical stress analysis, rock mechanics and fracture mechanics. Coupling these 
physical phenomena enables the study of all factors influencing the microwave-assisted rock breakage operation. Each of these factors may substantially change the process and its corresponding results.

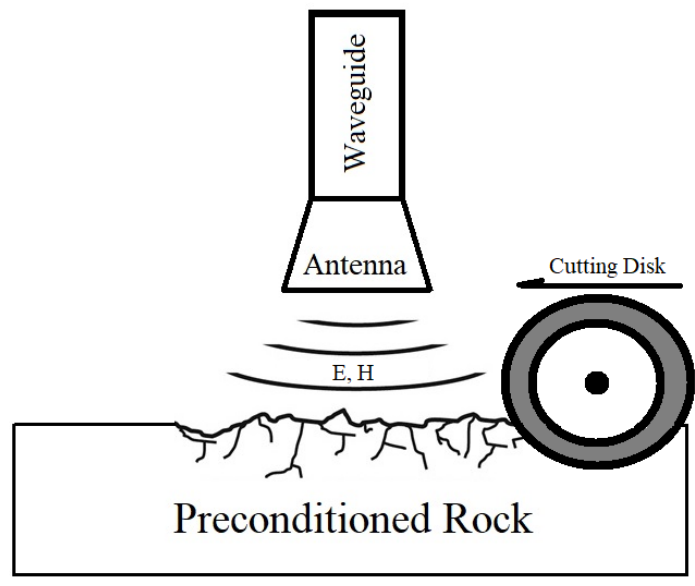

Fig. 1. The concept of microwave-assisted rock breakage

Over the past twenty years, several studies have highlighted the effects of microwave treatment on different rocks and minerals by performing both experimental tests and numerical simulations. Although several numerical studies have been done in recent years to investigate the effects of applied microwave irradiation on different rocks and minerals, most of the experiments were performed only to identify the potentiality and importance of microwave energy as an application in the rock breakage system. Also, none of the numerical simulations have focused on the effects of applying different operating parameters of microwave energy on thermal and mechanical responses of a rock model made up of differently attached rock samples under microwave exposure. The nature of the grain boundaries between the rock samples is not well understood, but it has been suggested that it is an area of disorder between two ordered species which can be considered as a potential area of weakness [4].

Since the rock breakage process by microwave energy depends mainly on different microwave operating parameters, a numerical modeling approach can easily evaluate the corresponding effects of the applied microwave, i.e. temperature gradients and initiation of mechanical stresses. Evaluation of these effects enables understanding of (1) how microwave energy affects minerals, and (2) what the effects of different microwave operating parameters on rock preconditioning are. Therefore, following the recent development of a fully coupled FE modeling approach by K. Teimoori et. al, [5], the current study uses the validated FE modeling approach to simulate a rock model consisting of a conceptual block of R1 host rock and R2 ore sample under microwave treatments with different operating parameters. The numerical models of the present study investigate microwave heating, thermal conduction, and thermally induced mechanical stresses on the boundaries of the two attached conceptual rock samples. Finally, the results of temperature and mechanical stress distribution are analyzed and compared with respect to different microwave input operating parameters.

The current study is part of an ongoing project that began in 2005 in the Geomechanics Laboratory at McGill University. The project's goal was to evaluate the influence of microwave irradiation with different operating parameters on different hard rocks. Ultimately, the objective of the project is to develop a new rock fracturing technique referred 
to as "non-explosive continuous rock breakage operation", where microwave energy is radiated from a pyramidal open-ended horn antenna onto the rock surface.

\section{Methodology}

Single-mode microwave cavities consist of a metallic enclosure and a waveguide horn. These metallic parts were designed so that the electromagnetic field polarization from a microwave signal will undergo several reflections. The superposition of the propagated waves in different directions gives rise to a standing wave pattern [6]. This pattern is very well defined inside the cavity's space. Consequently, the precise knowledge of different configurations of the electromagnetic waves enables the dielectric material (rock model) to be placed in the position at which maximum heating rate and subsequent maximum mechanical stress can be achieved. With that information in mind, a single-mode microwave cavity was chosen for numerical simulations in the present study. Therefore, the rock models of this study were exposed to microwaves with a frequency of $2.45 \mathrm{GHz}$ at $3 \mathrm{KW}$ and $9 \mathrm{KW}$ power levels, and at 30 seconds and 60 seconds exposure times when the distance from the antenna changes from $5 \mathrm{~cm}$ to $10 \mathrm{~cm}$. After simulations, the results of temperature and mechanical stress distribution were obtained in the R1 and R2 samples.

\subsection{Model development}

Fig. 2 presents the modeled single-mode microwave cavity and the rock model. The R1 rock host is rectangular with side-length of $40 \times 40 \mathrm{~cm}$, and the R2 ore sample has side-length of $2 \times 2 \mathrm{~cm}$ and height of $37 \mathrm{~cm}$. The rock models are placed on samples of glass insulator with side-lengths of $30 \times 30 \mathrm{~cm}$ and different heights (depending upon distances from the antenna). The rock models were used to conduct surface treatments in a single-mode cavity and with unidirectional loading conditions.

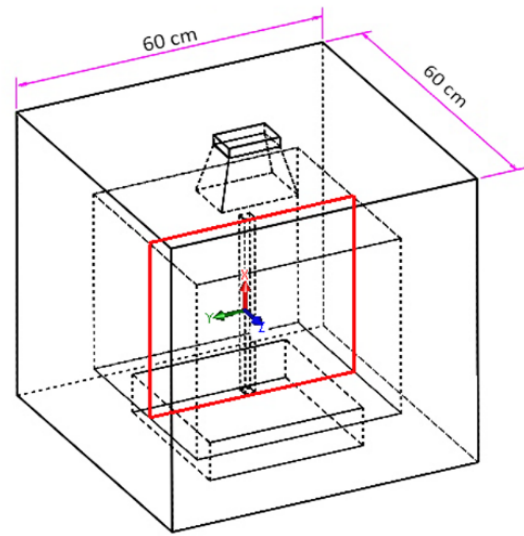

(a)

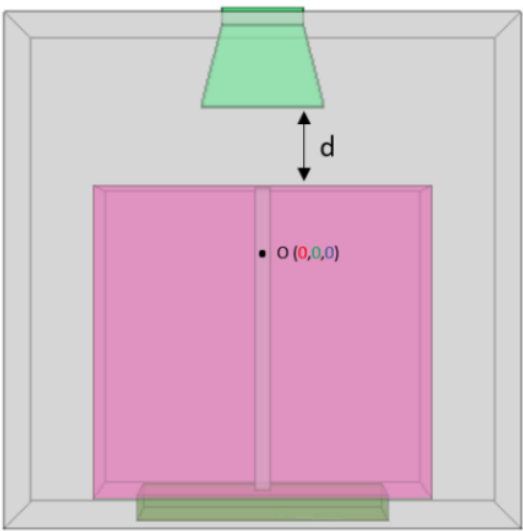

(b)

Fig. 2. The modelled single-mode microwave cavity including the R1 host rock and R2 ore sample, which is referred to as "rock model". (a) The 2D cut plane (shown by red) at the intersection of R1 and R2, and (b) the microwave equipment set-up with the rock model placed inside the microwave cavity and below the horn; the distance from the waveguide horn (antenna), $\mathrm{d}(\mathrm{cm})$, is shown by a black arrow. Here, the host rock is shown in pink, the ore sample in yellow, the glass insulator - beneath the rock model - in dark green, the waveguide horn in cyan, and the cavity walls in gray. 
As shown in Fig. 2, two different conceptual rock samples, R1 and R2, were assigned as the rock model inside the single-mode microwave cavity. The inclusion of these two different materials in the rock model was to investigate the effects of microwave treatments with different operating parameters on the boundaries between an ore sample and its surrounding rock host. To demonstrate the results of the simulations and to compare the obtained data with respect to different power levels, exposure times and distances from the antenna, a $2 \mathrm{D}$ cut plane at the intersection/boundary between the R1 and R2 samples was created for each simulation. This 2D cut plane is shown in Fig. 2a by red. By plotting the gradients of temperature and mechanical stress distribution on the 2D cut planes within the rock models, it will be possible to analyze and compare data with respect to the input microwave powers, distances from the antenna, and exposure times. In fact, the numerical models of two different materials presented in this paper can be considered an initial step to understand the mechanisms involved in such a complex system of rock-ore composition with three multiphysics interactions, and with respect to different microwave parameters.

\subsubsection{Governing equations}

Rocks are non-conductive materials usually made up of bonds of charged particles. The charged particles can absorb electricity when subjected to externally applied electromagnetic waves. In this case, fundamental electromagnetic theories apply and the solution to Maxwell's equations is required for this case study. Therefore, the problem of electromagnetic theory on a macroscopic level is that of solving Maxwell's equations subject to certain boundary conditions.

The amount of heat and mechanical stress produced in a rock sample when subjected to microwave treatment depends on the following parameters: power level, exposure time, and distance from the antenna (for single-mode microwave cavity type). The development of a numerical model to describe the electromagnetic-thermal-mechanical (ETM) multiphysics interaction in rocks requires careful consideration of the heating mechanism when microwave irradiation is applied. Therefore, it is important to express all three multiphysics mathematically.

Equations 1 to 3 were solved for electromagnetic, thermal and mechanical multiphysics to arrive at the final distribution of electric and magnetic fields, temperature, and maximum principal stress in the rock model as follows:

Electromagnetic waves $(\mathbf{E})$ :

$\nabla \times \mu_{r}^{-1}(\nabla \times \mathbf{E})-k_{0}^{2}\left(\epsilon_{r}-\frac{j \sigma}{\omega \epsilon_{0}}\right) \mathbf{E}=0, \quad k_{0}=\omega \sqrt{\varepsilon_{0} \mu_{0}}$

where,

E: is the electric field $(\mathrm{V} / \mathrm{m})$

$k_{0}$ : is the wave number $(\mathrm{rad} / \mathrm{m})$

$\mu_{r}:$ is the relative permeability

$\epsilon_{r}:$ is the relative permittivity

$\sigma:$ is the electrical conductivity

$\omega:$ is the angular frequency

$\varepsilon_{0} \& \mu_{0}$ : are the electric permittivity and magnetic permeability of free vacuum 
Heat transfer $(\mathbf{T})$ :

$\rho C_{P} \frac{\partial T}{\partial t}-\nabla \cdot(k \nabla \mathbf{T})=Q$

where,

$\rho:$ is the rock density $\left(\mathrm{kg} / \mathrm{m}^{3}\right)$

$C_{p}$ : is the heat capacity at constant pressure $\left(\mathrm{J} / \mathrm{kg}^{\circ} \mathrm{C}\right)$

$k$ : is the thermal conductivity $\left(\mathrm{W} / \mathrm{m}^{\circ} \mathrm{C}\right)$

$Q:$ is the heat source $\left(\mathrm{W} / \mathrm{m}^{3}\right)$

Solid mechanics (u):

$\rho \frac{\partial^{2} \mathbf{u}}{\partial t^{2}}=\mathbf{f}_{\mathrm{V}}-\nabla \cdot s$

where,

$\mathbf{f}_{\mathrm{V}}$ : is the deformation force $(N)$

$s:$ is the Cauchy stress $\left(N / m^{2}\right)$

\subsection{Numerics}

The finite element (FE) method is used to calculate the transient temperature, electromagnetic fields, and the mechanical stress/damage profiles in the rock models. The final mode, given by equations (1) to (3), together with appropriate boundary conditions, constitutive relations, and input material properties of the rock samples given in Table 1 constitute a highly coupled nonlinear differential algebraic system for the dependent variables $\mathrm{E}, \mathrm{T}$, and $\mathrm{u}$.

\begin{tabular}{ccc}
\hline Property name & $\mathrm{R} 2$ & $\mathrm{R} 1$ \\
\hline Thermal conductivity $(\mathrm{W} / \mathrm{m} \cdot \mathrm{K})$ & $37.9[4]$ & $1.55[5]$ \\
Heat capacity at constant pressure $(\mathrm{J} /(\mathrm{kg} \cdot \mathrm{K}))$ & $517[4]$ & $900[5]$ \\
Density $\left(\mathrm{kg} / \mathrm{m}^{3}\right)$ & $5018[4]$ & $2870[5]$ \\
Coefficient of thermal expansion $(1 / \mathrm{K})$ & $2.95 \mathrm{e}-6[7]$ & $5.4 \mathrm{e}-6[8]$ \\
Poisson's ratio $(1)$ & $0.16[4]$ & $0.28[5]$ \\
Young's modulus $(\mathrm{GPa})$ & $147[9]$ & $73[5]$ \\
Electrical conductivity $(\mathrm{S} / \mathrm{m})$ & $0.01[10]$ & $0.00005[11]$ \\
Relative permeability $(1)$ & $0.9-0.4 \mathrm{j}[12]$ & $1[5]$ \\
Relative permittivity $(1)$ & $7.6-1.3528 \mathrm{j}[12]$ & $7.77-0.85 \mathrm{j}[5]$ \\
\hline
\end{tabular}

Table 1. Input data of electrical, thermal, and mechanical properties of R1 and R2 samples for numerical models

The commercial FE solver, COMSOL multiphysics, was chosen to implement the derived models because of its versatility in handling general coupled nonlinear partial differential equations for fully coupled ETM multiphysics interaction. For time discretization, the backward differentiation formula (BDF) solver was employed as an implicit solver that uses backward differentiation formulas with second order of accuracy. The MUltifrontal Massively Parallel Sparse (MUMPS) direct solver was also employed as a sparse solver that is optimized for solving the system of equations in parallel, as this solver offers additional options for customization of parallel computing. A mesh-independent solution was ensured by comparing with results obtained using a coarse mesh consisting of 7,278 boundary elements, followed by several mesh adaptations until the difference in computed solid material's temperature was below $2 \%$, with a final mesh size of 7,492 . The geometries for all numerical models were resolved for 1,438,592 number of degrees of freedom (DOFs), plus 12,158 internal DOFs, to ensure mesh independent solutions. The computations carried out on a $3.5 \mathrm{GHz}$ PC with $128 \mathrm{~GB}$ RAM, required around $\sim 6047 \mathrm{~s}$ (1 hour, 40min, $47 \mathrm{~s}$ ) for the simulations. 


\section{Results and Discussion}

For better visual understanding of the numerical models' results, a 3D distribution of the electric field, temperature, and maximum principal stress is plotted when microwave irradiation of $3 \mathrm{KW}$ power, 30 seconds exposure, and $5 \mathrm{~cm}$ distance from the antenna is applied to a rock model (see Fig. 3).

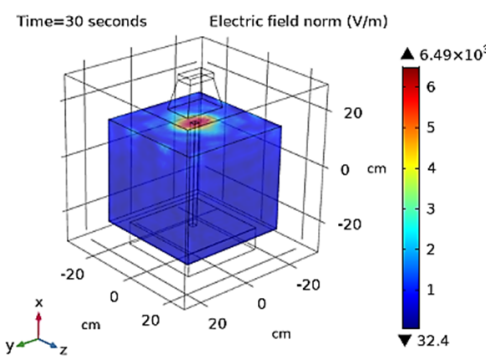

(a)

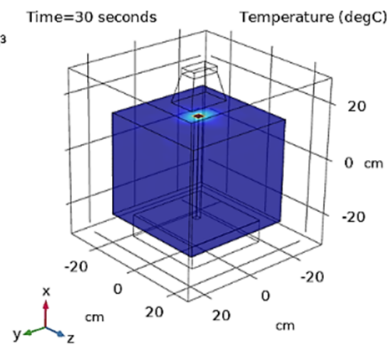

(b)

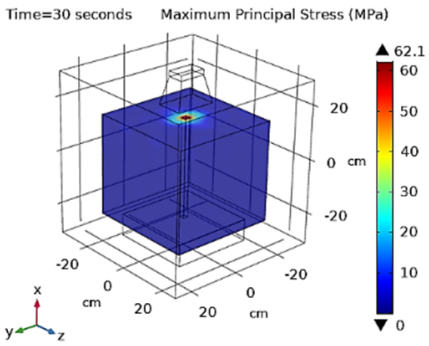

(c)

Fig. 3. Three-dimensional distribution of (a) electric field $(\mathrm{V} / \mathrm{m}),(\mathrm{b})$ temperature $\left({ }^{\circ} \mathrm{C}\right)$, and (c) maximum principal stress $(\mathrm{MPa})$ in a rock model when microwave irradiation of $3 \mathrm{KW}$ is applied at $5 \mathrm{~cm}$ distance from the antenna for 30 seconds exposure

As can be seen in Fig. 3, the distribution of temperature and maximum principal stress gradients differs between the R1 and R2 samples. To illustrate this phenomenon, a 2D cut plane was made at the coordinate of $Z=1 \mathrm{~cm}$, where the distributions occur on the boundaries between the two rock samples. It should be noted that rocks are usually anisotropic and consist of different minerals; therefore, the distribution of temperature and mechanical stresses on the boundaries of the two different rock samples varies depending upon their material properties and the amount of applied microwave irradiation. These results are shown in Figs. 4 and 5.

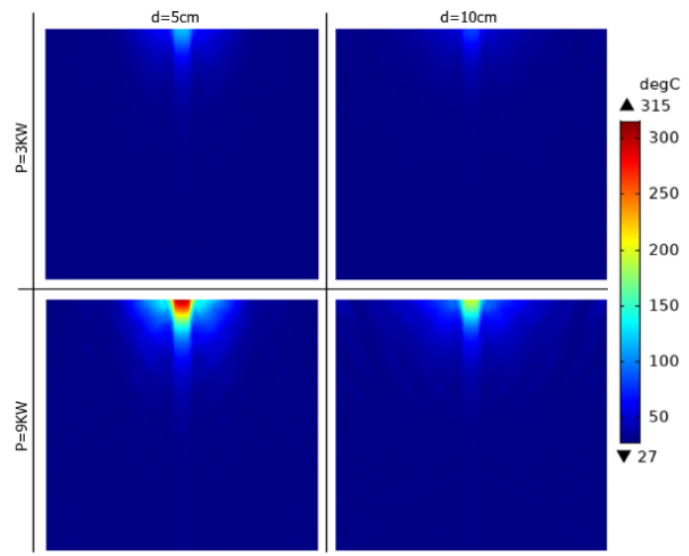

(a) $\mathrm{t}=30$ seconds

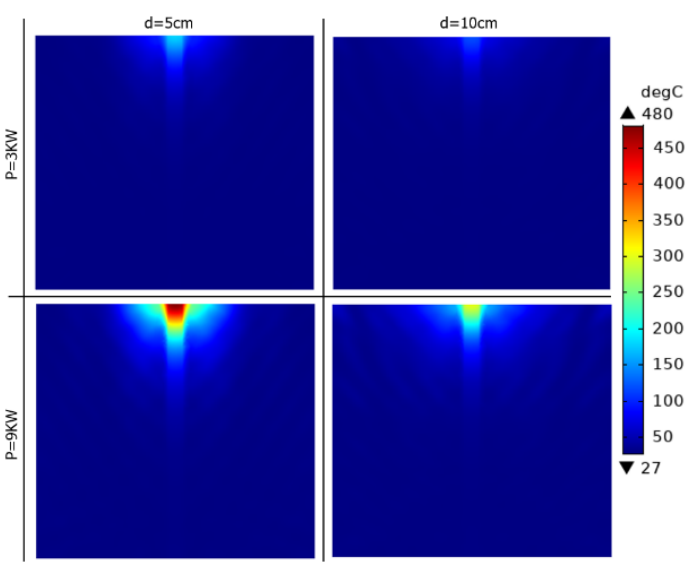

(b) $\mathrm{t}=60$ seconds

Fig. 4. Two-dimensional temperature distribution for power levels of $3 \mathrm{KW}$ (top) and $9 \mathrm{KW}$ (bottom), and distances of $5 \mathrm{~cm}$ (left) and $10 \mathrm{~cm}$ (right) at (a) $\mathrm{t}=30$ seconds and (b) $\mathrm{t}=60$ seconds exposure 
The temperature distribution in Fig. $4 \mathrm{a}$ and $4 \mathrm{~b}$ has a direct relationship to the microwave power. This means that by increasing power, a rise in temperature is observed; however, the distance from the antenna decreases the temperature. As the distance from the antenna increases from $5 \mathrm{~cm}$ to $10 \mathrm{~cm}$, the temperature distribution becomes lower. A higher distance from the antenna distributes the patterns of temperature more evenly. The exposure time is also considered to play a substantial role in the heating process; as expected, an increase in exposure time can result in higher temperature and mechanical stress values. This can be verified by comparing Fig. 4a with Fig. 4b, and Fig. 5a with Fig. 5b, when exposure time increases from 30 seconds to 60 seconds.

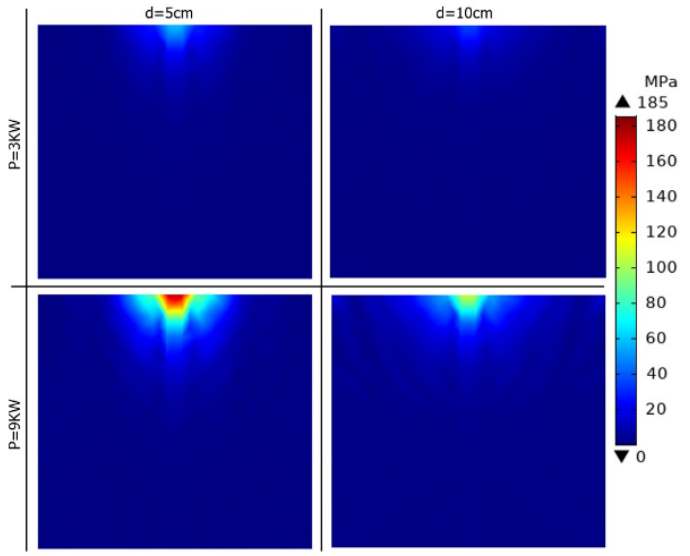

(a) $\mathrm{t}=30$ seconds

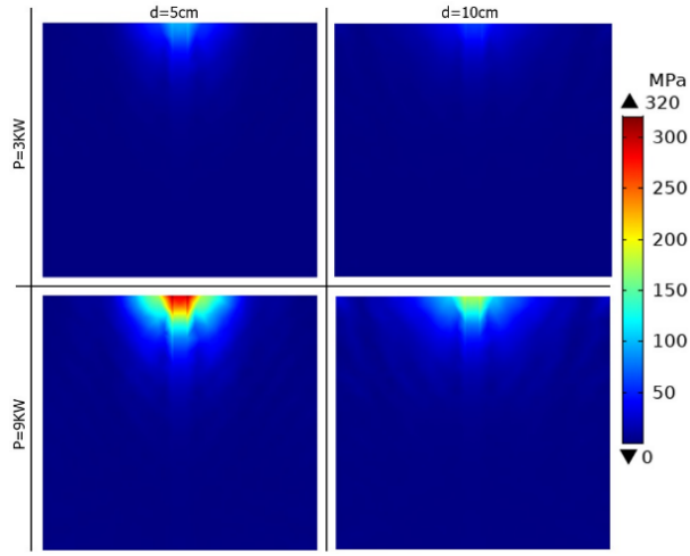

(b) $\mathrm{t}=60$ seconds

Fig. 5. Two-dimensional maximum principal stress distribution for power levels of $3 \mathrm{KW}$ (top) and 9KW (bottom), and distances of $5 \mathrm{~cm}$ (left) and $10 \mathrm{~cm}$ (right) at (a) $\mathrm{t}=30$ seconds and (b) $\mathrm{t}=60$ seconds exposure

A pattern of simulated maximum principal stresses developed within the modelled R2 ore sample inside the R1 host rock after simulations is shown in Fig. 5. The results show that the maximum principal stress occurred very close to the grain boundary between R1 and R2 samples. Also, as the distance from the antenna increases, the intensity of the maximum principal stress decreases. Consequently, there is an inverse relationship between the power level and the distance from the antenna. Furthermore, as the power level increases, the intensity of maximum principal stress increases. Increasing the power level from $3 \mathrm{KW}$ to $9 \mathrm{KW}$ provided a sharper and more distributed mechanical stress gradients. It should be noted that when the mechanical stress exceeds the strength of the material, then plastic deformation and crack propagation begins. In the current study, the highest value of maximum principal stress achieved $(315 \mathrm{MPa})$ was when $9 \mathrm{KW}$ power was applied for 60 seconds exposure. This value should be considered a failure given that rocks generally fail at approximately $240 \mathrm{MPa}$ (see ref [5]). By comparing Fig. 5a with Fig. 5b for the same $(\mathrm{P}=9 \mathrm{KW}$ and $\mathrm{d}=5 \mathrm{~cm})$ configuration, the highest value of maximum principal stress achieved was $174 \mathrm{MPa}$ and $315 \mathrm{MPa}$. This means that for a high power level, the exposure time needed to cause thermally induced microcracking, and subsequent breakage, is shorter and the energy is very efficiently used to produce breakage. This finding is very consistent with the results from [5]. A higher input power level of microwaves contributes to more complex physical and mechanical reactions, which include the elevation of internal pressure and decomposition of particles along the boundary between the R1 host and the R2 ore sample. This can be thought of as the fundamental mechanism of cracking in rocks composed of different materials; as 
thermal stresses increase, unstable growth of cracks along the boundaries of different minerals will be observed.

\section{Conclusion}

In this paper, the thermally induced mechanical stresses on the boundaries of two conceptual attached rock samples are numerically simulated by the finite element method, and the dependence of the failure mechanism on microwave input operating parameters is studied. The present study shows that differential volumetric expansion of a rock model, which includes a host rock and an ore sample, during microwave heating increases mechanical stresses along grain boundaries. Also, exposure time is a parameter that can influence this phenomenon; more exposure time will result in more mechanical stresses and subsequent cracking/spallation.

The results from the current study can be thought of as groundwork for the future of microwave energy application in civil and mining engineering related to rock breakage. The numerical results suggest that microwave energy has a significant application for rock breakage systems and, therefore, for continuous excavation machinery. In terms of modelling and simulation, this work provides some tools and insights into the design and performance of microwave-assisted heating of a rock model that is made up of two attached types of rocks.

\section{Acknowledgements}

Financial support for this work from the Natural Sciences and Engineering Research Council (NSERC) of Canada, the McGill Engineering Doctoral Award (MEDA) Award is gratefully acknowledged.

\section{References}

[1] Lindroth, D. P., Morrell, R. J., and Blair, J. R., "Microwave Assisted Hard Rock Cutting”, U.S. Patent, No. 5,003,144. 26 Mar. 1991.

[2] Hassani, F., Nekoovaght, P. M., and Gharib, N., "The Influence of Microwave Irradiation on Rocks for Microwave-Assisted Underground Excavation”, J. Rock Mech. Geotech. Eng., 2016, 8(1), pp. 1-15.

[3] Lu, G. ming, Li, Y. hui, Hassani, F., and Zhang, X., "The Influence of Microwave Irradiation on Thermal Properties of Main Rock-Forming Minerals”, Appl.

Therm. Eng., 2017, 112, pp. 1523-1532.

[4] Wang, Y., and Djordjevic, N., "Thermal Stress FEM Analysis of Rock with Microwave Energy”, Int. J. Miner. Process., 2014, 130, pp. 74-81.

[5] Teimoori, K., Hassani, F., and Sasmito, A. P., "Multiphysics Study of Microwave Irradiation Effects on Rock Breakage System”, IJRMMS, 2019, 1(514), pp. 1-32.

[6] Whittles, D. ., Kingman, S. ., and Reddish, D., "Application of Numerical Modelling for Prediction of the Influence of Power Density on Microwave-Assisted Breakage”, Int. J. Miner. Process., 2003, 68(1-4), pp. 71-91.

[7] Press, D. C., "Thermal Expansion of Fluorspar and Iron Pyrite”, Proc. Indian Acad. Sci. - Sect. A, 1949, 30(6), pp. 284-294.

[8] Hartlieb, P., Toifl, M., Kuchar, F., Meisels, R., and Antretter, T., "ThermoPhysical Properties of Selected Hard Rocks and Their Relation to MicrowaveAssisted Comminution”, Miner. Eng., 2016, 91, pp. 34-41.

[9] Whitaker, M. L., Liu, W., Wang, L., and Li, B., "Acoustic Velocities and Elastic Properties of Pyrite (FeS 2) to 9.6 GPa”, J. Earth Sci., 2010, 21(5), pp. 792-800.

[10] Pearce, C. I., "Electrical and Magnetic Properties of Sulfides", Rev. Mineral. Geochemistry., 2006, 61(1), pp. 127-180. 
[11] Carmichael, R. S., "Practical Handbook of Physical Properties of Rocks and Minerals", CRC press, 2018.

[12] Peng, Z., Hwang, J.-Y., Kim, B.-G., Kim, J.-Y., and Wang, X., "Microwave Permittivity, Permeability, and Penetration Depth of Pyrite”, Characterization of Minerals, Metals, and Materials., 2014, pp. 371-378. 